\title{
RECUPERAÇÃO DE SÍLICA-GEL UTILIZANDO PROCESSOS OXIDATIVOS AVANÇADOS: UMA ALTERNATIVA SIMPLES E DE BAIXO CUSTO
}

Silvio César Godinho Teixeira, Leda Mathias e Maria Cristina Canela*

Laboratório de Ciências Químicas, Centro de Ciência e Tecnologia, Universidade Estadual do Norte Fluminense, Av. Alberto Lamego, 2000, 28013-602 Campos dos Goytacazes - RJ

Recebido em 26/8/02; aceito em 31/3/03

\begin{abstract}
SILICA GEL RECYCLING USING AND ADVANCED OXIDATIVE PROCESS: A SIMPLE AND LOW-COST ALTERNATIVE. Silica gel is widely used as adsorbent for isolating and purifying natural compounds. Intensive use and high cost make this process expensive and generate solid residues contaminated with many different organic compounds. In the present work a simple method for recycling silica was investigated, by using Advanced Oxidative Processes. Silica gel was treated with $\mathrm{H}_{2} \mathrm{O}_{2}$ /solar light and compared with a sample treated by conventional methods (high temperature and oxidation with $\mathrm{KMnO}_{4}$ ). High temperature treatment changes the structure of the silica and, consequently, the separation efficiency. Oxidation by using $\mathrm{KMnO}_{4}$ requires multiple steps and produces residues, including manganese and oxalic acid. The method using $\mathrm{H}_{2} \mathrm{O}_{2}$ /solar light to recuperate silica gel does not modify its separation efficiency and is less expensive than the traditional methods. Additionally, HPLC and GC-MS analysis indicate that $\mathrm{H}_{2} \mathrm{O}_{2}$ /solar light eliminates all residues of the silica gel.
\end{abstract}

Keywords: advanced oxidative process; recycling; silica gel.

\section{INTRODUÇÃO}

A sílica-gel é um dos principais adsorventes utilizados no isolamento e purificação de compostos em laboratórios que trabalham com produtos naturais. A sílica-gel, usada nestes laboratórios de modo intensivo, possui um alto custo e gera um resíduo sólido contaminado com compostos orgânicos. Nesse contexto, metodologias de recuperação da sílica-gel são propostas na literatura ${ }^{1,2}$. Estas metodologias incluem a oxidação com $\mathrm{KMnO}_{4}$ em meio ácido ${ }^{2} \mathrm{e}$ com $\mathrm{H}_{2} \mathrm{O}_{2}$ em meio ácido ${ }^{2}$ e tratamento térmico por calcinação em altas temperaturas ${ }^{1}$.

Os processos oxidativos avançados têm sido uma alternativa de grande interesse para o tratamento de matrizes contaminadas com compostos orgânicos. Estes processos são mais intensivamente utilizados para o tratamento de efluentes em fase aquosa e gasosa e, mais recentemente, têm sido aplicados para matrizes sólidas, como solos e sedimentos ${ }^{3-5}$. Os processos oxidativos geralmente envolvem geração de espécies altamente oxidantes e não seletivas, como o radical hidroxila $(\bullet \mathrm{OH})$ e, em alguns casos, o oxigênio singlete. $\mathrm{O}$ radical - OH pode ser gerado por processos fotoquímicos ou não-fotoquímicos para oxidar contaminantes no ambiente, convertendo-os em espécies inócuas.

Tendo em vista a implementação de um programa de tratamento de resíduos no Laboratório de Ciências Químicas da UENF, a recuperação da sílica-gel gerada como resíduo sólido é um dos pontos a serem trabalhados neste programa. O Setor de Produtos Naturais gera cerca de $1 \mathrm{~kg}$ de sílica por mês, tendo acumulado um total de 20 $\mathrm{kg}$ de sílica na forma de resíduo sólido, passível de tratamento e de difícil descarte. Assim, o objetivo deste trabalho é estudar uma metodologia simples, de baixo custo e alta eficiência para recuperação da sílica-gel através de processos oxidativos avançados e realizar uma comparação com as outras metodologias propostas na literatura.

\footnotetext{
*e-mail: mccanela@uenf.br
}

\section{PARTE EXPERIMENTAL}

A sílica-gel utilizada para purificação e separação de extratos no Setor de Produtos Naturais é originalmente a Silicagel 60 (Merck) de granulometria entre 70 a 230 mesh $(0,063$ - 0,200 nm). Em cada ensaio foram utilizadas amostras de $100 \mathrm{~g}$ de resíduo de sílica-gel, recolhidas de um mesmo lote, que foram tratadas como segue abaixo:

$\mathrm{S} 2$ - adição de $125 \mathrm{~mL}$ de $\mathrm{H}_{2} \mathrm{O}_{2} 30 \%$ e $125 \mathrm{~mL}$ de água destilada ;

$\mathrm{S} 3$ - adição de duas alíquotas de $65 \mathrm{~mL}$ de $\mathrm{H}_{2} \mathrm{O}_{2} 30 \%$, com intervalo de $1 \mathrm{~h}$, sem adição de água;

S6- idem ao S3, com exposição ao sol durante 5 h no período das 10 às $15 \mathrm{~h}$, em uma placa de Petri;

S7-idem ao 66 , com aquecimento em mufla a $800{ }^{\circ} \mathrm{C}$ por $2 \mathrm{~h}$;

S8- lavagem com $50 \mathrm{~mL}$ de metanol, seguida de calcinação em mufla por $6 \mathrm{~h}$ a $800{ }^{\circ} \mathrm{C}$.

S9- lavagem com $200 \mathrm{~mL}$ de etanol, seguida da adição de $20 \mathrm{~mL}$ de $\mathrm{KMnO}_{4}$ 0,1 mol L-1 em meio ácido. Após a reação do $\mathrm{KMnO}_{4}$, foram adicionados $2 \mathrm{~g}$ de ácido oxálico para eliminar o excesso do reagente e a sílica foi lavada com água destilada até que o meio se tornasse neutro.

Os tratamentos S2, S3 e S9 foram baseados no trabalho de Rieghl e Pinto ${ }^{2}$ e o tratamento S8, no trabalho de Loureiro e colaboradores ${ }^{1}$. As amostras S6 e S7 foram expostas à irradiação solar utilizando placas de Petri de área $308 \mathrm{~cm}^{2}$. Para eliminar o efeito do calor provocado pela irradiação solar, as amostras submetidas aos tratamentos na ausência de luz (S2 e S3) foram colocadas juntamente com as outras amostras, sob o sol, porém protegidas por um filme de alumínio. Durante o tratamento, as amostras foram periodicamente homogeneizadas com um bastão de vidro e água foi adicionada para evitar a secagem da sílica.

Após os tratamentos, as amostras foram filtradas e lavadas com $200 \mathrm{~mL}$ de água destilada e ativadas por $12 \mathrm{~h}$ a $120^{\circ} \mathrm{C}$. A presença de peróxido de hidrogênio residual foi verificada através da reação com metavanadato de amônio e posterior análise espectrofotométrica em $446 \mathrm{~nm}^{6}$. Foram utilizados $4 \mathrm{~mL}$ da solução sobrenadante da sílica, 
$2 \mathrm{~mL}$ de metavanadato de amônio, completando-se o volume com água desionizada para $10 \mathrm{~mL}$.

As amostras de sílica-gel foram lavadas com diclorometano, hexano e metanol. Os resíduos da lavagem com diclorometano e hexano foram injetados em cromatógrafo a gás acoplado a um espectrômetro de massas (CG-EM), Shimadzu QP5050A, equipado com uma coluna DB-1. Do resíduo da lavagem com metanol, uma alíquota de $20 \mu \mathrm{L}$ foi injetada em aparelho de cromatografia líquida de alta eficiência (CLAE), Shimadzu SPD-M10AVP, equipado com uma coluna Lichrospher ${ }^{\circledR} 100$ RP-18 (5 $\left.\mu, 250-4\right)$ e um detector espectrofotométrico de arranjo de diodos, empregando-se o sistema de gradiente linear de $100 \%$ metanol e um fluxo de $0,7 \mathrm{~mL}$ por minuto e detecção a $280 \mathrm{~nm}$. O resíduo da lavagem em metanol também foi analisado em espectrofotômetro de UV-Vis, Shimadzu UV$1601 \mathrm{PC}$, na faixa de $200-800 \mathrm{~nm}$.

A modificação da estrutura superficial da sílica após os diversos tratamentos foi avaliada utilizando um aparelho de medida de área superficial Quantachrome Autosorb 1-C. A eficiência de separação foi avaliada através de cromatografia de coluna, onde foram pesados $35 \mathrm{~g}$ de sílica-gel tratada, utilizando-se um extrato de espinafre que foi eluído com acetona e éter de petróleo na proporção (30:70). Finalmente, realizou-se uma análise de custo das diversas metodologias de tratamento.

\section{RESULTADOS E DISCUSSÃO}

$\mathrm{O}$ peróxido de hidrogênio é um oxidante $\left(\mathrm{E}_{\text {oxidação }}=1,77 \mathrm{eV}\right)$ utilizado para destruição de compostos orgânicos. Contudo, sob irradiação este reagente promove a formação de radicais hidroxilas que são oxidantes mais fortes $\left(\mathrm{E}_{\text {oxidação }}=2,80 \mathrm{eV}\right)^{7}$. Segundo Huang e colaboradores ${ }^{8}$, e Legrini e colaboradores ${ }^{9}$, o mecanismo aceito para a fotólise de $\mathrm{H}_{2} \mathrm{O}_{2}$ é a quebra da molécula em radicais hidroxila, com um rendimento de dois $\bullet \mathrm{OH}$ para cada molécula de $\mathrm{H}_{2} \mathrm{O}_{2}$ (Equação 1).

$\mathrm{H}_{2} \mathrm{O}_{2}+\mathrm{hv} \rightarrow 2 \cdot \mathrm{OH}$

Os resultados obtidos neste trabalho comprovaram a maior eficiência no tratamento da sílica-gel com $\mathrm{H}_{2} \mathrm{O}_{2} /$ luz solar (S6). Nos resíduos de lavagem da sílica com diclorometano e hexano, obtidos após os diversos métodos de tratamento, não foi observada a presença de resíduos de substâncias apolares e de polaridade média, quando injetados no CG-EM. Os espectros de absorção dos resíduos de lavagem das diversas sílicas com metanol mostraram que as amostras tratadas apenas com $\mathrm{H}_{2} \mathrm{O}_{2}(\mathrm{~S} 2, \mathrm{~S} 3)$ ou $\mathrm{KMnO}_{4}$ (S9) foram as que apresentaram maiores absorções. As amostras que apresentam menor absorção foram S6, S7 e S8 (Figura 1). Para S7 e S8, este resultado era esperado, uma vez que as amostras foram submetidas a $800^{\circ} \mathrm{C}$, temperatura que destrói toda a matéria orgânica adsorvida na sílica. A ausência de absorção na S6 demonstra a eficiência do processo de tratamento proposto. Os resultados obtidos quando estas amostras foram injetadas no CLAE (Figura 2) confirmam a presença de várias impurezas na sílica S2. Assim, as amostras que apresentaram menos resíduos aderidos à sílica após o tratamento foram S6, S7, S8 e S9.

A área superficial da sílica-gel foi verificada para comparar mudanças após tratamento com altas temperaturas. Os resultados estão apresentados na Tabela 1, onde se observa maior diminuição na área superficial nas sílicas submetidas a temperaturas altas (S7 e S8). Esta diminuição está relacionada com a perda de água, através da condensação dos grupos silanóis para siloxanos, alterando-se assim a superfície da mesma9 .

A cromatografia de coluna utilizando extrato de espinafre mos-

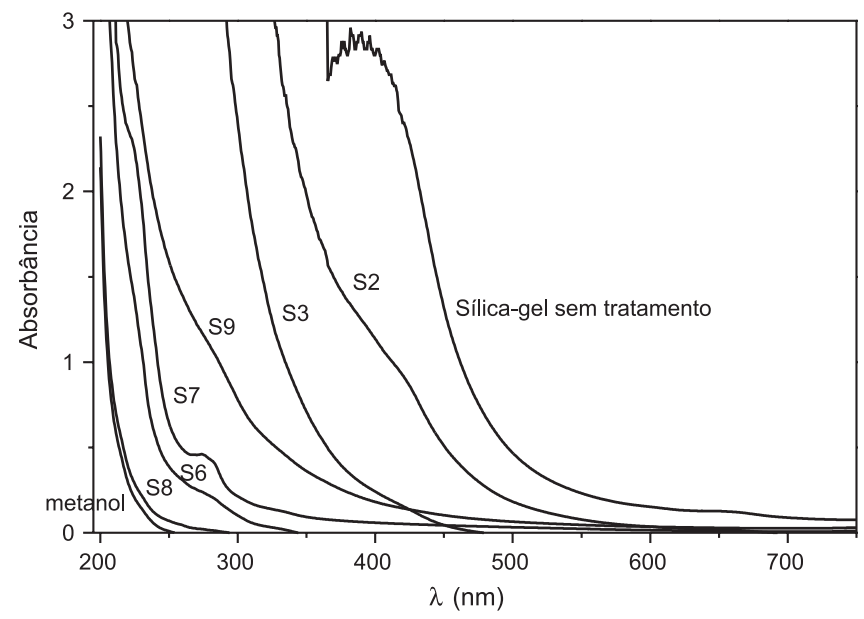

Figura 1. Espectro dos extratos de metanol analisados por espectrofometria de UV-Vis

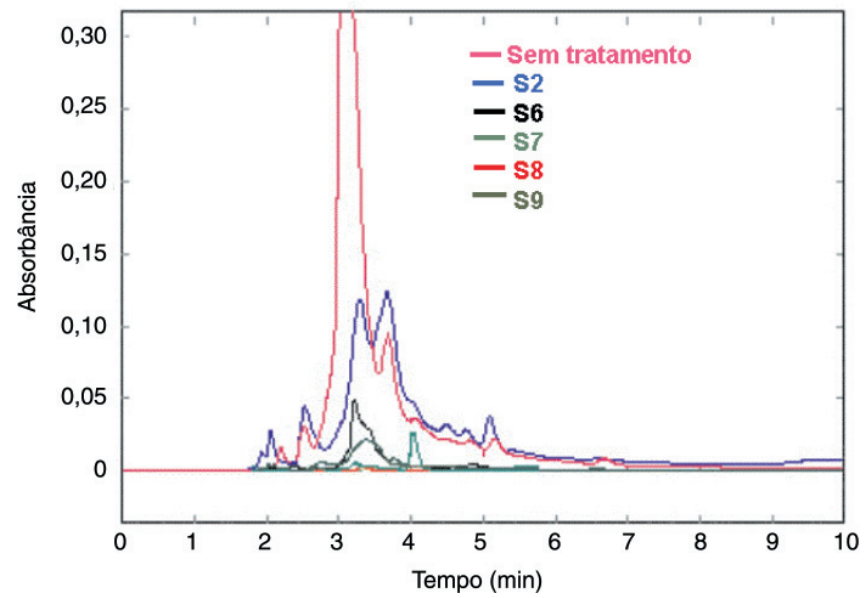

Figura 2. Cromatograma dos extratos de metanol obtidos através de CLAE

trou que as sílicas tratadas com as metodologias S6 e S9 foram as que apresentaram a melhor separação dos componentes: clorofila e $\beta$-caroteno (Figura 3). As sílicas tratadas termicamente mostraramse ineficientes para promover a separação destes componentes.

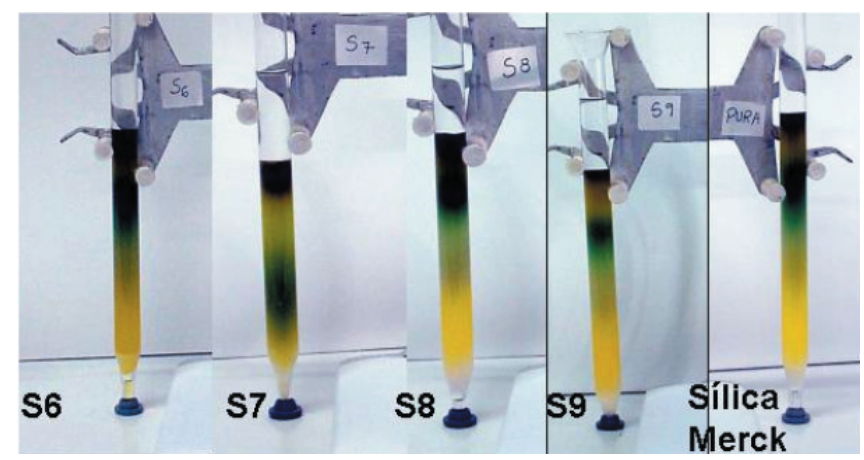

Figura 3. Ensaio de separação dos componentes do extrato de espinafre utilizando as sílicas tratadas

Finalmente, através de uma análise de custos (Tabela 1) é possível verificar que a reciclagem da sílica-gel, independente da metodologia utilizada, é muito vantajosa em comparação com o pre- 
Tabela 1. Área superficial da sílica-gel antes e após os tratamentos e custo comparativo dos vários tratamentos

\begin{tabular}{lcccccc}
\hline Amostra & $\begin{array}{c}\text { Sílica-gel } \\
\text { 60 Merck }\end{array}$ & S2 & S6 & S7 & S8 & S9 \\
\hline BET $\left(\mathrm{m}^{2} / \mathrm{g}\right)$ & 341,7 & 299,9 & 293,3 & 270,5 & 269,9 & 310,0 \\
Custo (US $\$ / \mathrm{kg})$ & 125,00 & 5,50 & 5,50 & 5,50 & 16,00 & 9,00 \\
\hline
\end{tabular}

ço da mesma pura. No entanto, quando comparamos as metodologias entre si, podemos observar que todo processo envolvendo altas temperaturas acumula um custo energético muito alto, além de que os fornos tipo mufla, existentes nos laboratório de pesquisa, normalmente são pequenos e capazes de tratar apenas reduzidas quantidades de material. Os processos utilizando oxidantes são de mais baixo custo. A utilização de peróxido de hidrogênio sem irradiação solar é possível, como demonstrado por Riehl e Pinto $^{2}$, e no experimento S2. No entanto, para a remoção total dos contaminantes, é necessária uma maior quantidade de peróxido de hidrogênio em comparação ao sistema irradiado com luz solar.

Neste trabalho, a remoção total do contaminante da sílica utilizando peróxido de hidrogênio e irradiação solar (S6) foi realizada em 5 h de exposição, no período do dia entre 10 e 15 h, com céu claro, durante o mês de dezembro. A latitude da cidade de Campos dos Goytacazes é de $21^{\circ} 45^{\prime} 15^{\prime \prime} \mathrm{Sul}^{11}$. Este período de exposição foi suficiente para que todo o peróxido de hidrogênio fosse consumido. Estes dados são importantes, uma vez que o tempo de exposição para remoção total de contaminantes pode variar de acordo com o período do dia, condições do tempo e latitude ${ }^{12}$. Para volumes maiores de material a ser tratado, um tempo de exposição maior pode ser requerido. A utilização de luz artificial, com maior energia (e.g. luz germicida), pode ser uma alternativa para acelerar o processo de remoção, embora acrescente um custo adicional e a necessidade de aparatos para iluminação. $\mathrm{O}$ objetivo deste trabalho foi propor uma alternativa de baixo custo e simplicidade de implementação na recuperação da sílica-gel.

\section{CONCLUSÕES}

A comparação entre as diversas metodologias empregadas no tratamento de sílica-gel indica que a utilização de peróxido de hidrogênio e irradiação solar (S6) é um tratamento simples, não altera a superfície da sílica, remove com eficiência as impurezas presentes na mesma e é de baixo custo. Neste tratamento também não foi observada a presença de $\mathrm{H}_{2} \mathrm{O}_{2}$ residual após a irradiação. $\mathrm{O}$ tratamento com mufla (S8) elimina os contaminantes da sílica com eficiência, porém, modifica as propriedades de separação da sílica-gel, além de possuir um alto custo. A metodologia S9 também se mostrou eficiente na remoção de substâncias orgânicas da sílica, a qual não é alterada quanto a sua seletividade, e não tem um alto custo. No entanto, a utilização desta metodologia envolve uma série de etapas e geração de resíduos de Mn e ácido oxálico.

\section{REFERÊNCIAS}

1. Loureiro, A. P.; Souza, J. A.; Aparecido, D.; Fernandes, J. B.; Quim. Nova 1991, 14, 112.

2. Riehl, C. A. S.; Pinto, A. C.; Quim. Nova 1988, 11, 329.

3. Chiron, S.; Fernandez-Alba, A.; Rodriguez, A.; Garcia-Calvo, E.; Water Res. 2000, 34, 366.

4. Nogueira, R. F. P.; Guimarães, J. R.; Revista de Engenharia Sanitária e Ambiental 1998, 3, 97.

5. Higarashi, M. M.; Jardim, W. F.; Am. Lab. 2000, 32, 25.

6. Oliveira, M. C.; Nogueira, R. F. P; Neto, J. A. G.; Jardim, W. F.; Rohwedder, J. J. R.; Quim. Nova 2001, 24, 188.

7. U.S. Environmental Protection Agency; Handbook Advanced Photochemical Oxidation Processes (EPA/625/R-98/004), Cincinnati, E.U.A., 1998, p. 1-1.

8. Huang, C. P.; Dong, C.; Tang, Z.; Waste Manag. 1993, 13, 361.

9. Legrini, O.; Oliveros, E.; Braun, A. M.; Chem. Rev. 1993, 93, 671.

10. Scott, R. P. W.; Silica gel and Bonded Phases - Their Production, Properties and Use in LC., Ed. John Wiley \& Sons: Chichester, 1993.

11. http://www.aondefica.com/lat_3_.asp, acessada em Setembro 2003.

12. Nogueira, R. F. P.; Trovo, A. G.; Modé, D. F.; Chemosphere 2002, 48, 385. 